\title{
Appropriateness of Percutaneous Coronary Intervention Performed by Japanese Expert Operators in Patients With Chronic Total Occlusion
}

\author{
Tomotsugu Seki, MD; Hironobu Tokumasu, MD; Hiroyuki Tanaka, MD; \\ Harumi Katoh, MD, PhD; Koji Kawakami, MD, PhD
}

\begin{abstract}
Background: The appropriateness of percutaneous coronary intervention (PCI) for chronic total occlusion (CTO) lesions has rarely been investigated.

Methods and Results: The Japanese CTO-PCI Expert Registry enrolled consecutive patients undergoing CTO-PCl carried out by highly experienced Japanese CTO specialists who performed more than $50 \mathrm{CTO}$-PCls per year and $300 \mathrm{CTO}$-PCls in total. This study included patients undergoing CTO-PCI between January 2014 and December 2019. The appropriateness, trends, and differences among the procedures performed by the operators using the 2017 appropriate use criteria were analyzed. Furthermore, we performed a logistic regression analysis to assess whether the appropriateness was associated with in-hospital major adverse cardiovascular and cerebrovascular events (MACCE). Of the 5,062 patients who underwent $\mathrm{CTO}-\mathrm{PCl}, 4,309$ (85.1\%) patients who did not undergo the non-invasive stress test were classified as having no myocardial ischemia. Of the total cases, 3,150 (62.2\%) were rated as "may be appropriate," and $642(12.7 \%)$ as "rarely appropriate" CTO-PCI cases. The sensitivity analyses showed that the number (\%) of "may be appropriate" ranged from 4,125 (57.8\%) to 4,744 (66.4\%) and the number of "rarely appropriate" ranged from $843(11.8 \%)$ to $970(13.6 \%)$ among best and worst scenarios.
\end{abstract}

Conclusions: In a large Japanese CTO-PCI registry, approximately $13 \%$ of CTO-PCI procedures were classified as "rarely appropriate". Substantial efforts would be required to decrease the number of "rarely appropriate" CTO-PCI procedures.

Key Words: Appropriate use criteria; Chronic total occlusion; Percutaneous coronary intervention

C oronary chronic total occlusions (CTOs) are defined as occluded coronary segments with Thrombolysis in Myocardial Infarction flow grade 0 for $\geq 3$ months. CTOs are commonly encountered complex lesions identified in $18-52 \%$ of all patients undergoing coronary angiography..$^{1-3}$ In recent years, advances in technology and the use of new techniques have increased the procedural success rates and decreased the complication rates of percutaneous coronary intervention (PCI) for CTO lesions. ${ }^{4}$

In 2017, the American College of Cardiology and American Heart Association, along with other professional societies, updated the Appropriate Use Criteria (AUC) for Coronary Revascularization in Patients with Stable Ischemic Heart Disease (SIHD). The purpose was to critically examine and improve patient selection for coronary artery revascularization and address concerns about the potential overuse of PCI in patients with SIHD. ${ }^{5}$ The method of

\begin{abstract}
Editorial p ????
developing the AUC for coronary revascularization in SIHD patients has been previously described. ${ }^{5}$ The writing group created clinical scenarios that represent the most common clinical scenarios encountered in clinical practice. Using the modified Delphi approach, an expert panel member judged the appropriateness of the clinical scenarios. The AUC have been applied to real-world clinical practice along with data from various registries, and studies have demonstrated a strong possibility of PCI overuse in non-CTO-PCI cases in real-world practice. ${ }^{6-10}$

In addition, recent randomized control trials (RCTs) showed only modest effects of CTO-PCI. An open-label, multicenter RCT (Drug-Eluting Stent Implantation vs. Optimal Medical Treatment in Patients with Chronic
\end{abstract}

Received June 10, 2021; revised manuscript received August 26, 2021; accepted August 28, 2021; J-STAGE Advance Publication released online October 6, 2021 Time for primary review: 13 days

Department of Pharmacoepidemiology, Graduate School of Medicine and Public Health, Kyoto University, Kyoto (T.S., K.K.); Department of Cardiovascular Medicine, Graduate School of Medical Science, Kyoto Prefectural University of Medicine, Kyoto (T.S.); and Kurashiki Central Hospital, Kurashiki (H. Tokumasu, H. Tanaka, H.K.), Japan

Mailing address: Koji Kawakami, MD, PhD, Department of Pharmacoepidemiology, Graduate School of Medicine and Public Health, Kyoto University, Yoshida Konoe-cho, Sakyo-ku, Kyoto 606-8501, Japan. E-mail: kawakami.koji.4e@kyoto-u.ac.jp

All rights are reserved to the Japanese Circulation Society. For permissions, please e-mail: cj@j-circ.or.jp

ISSN-1346-9843 


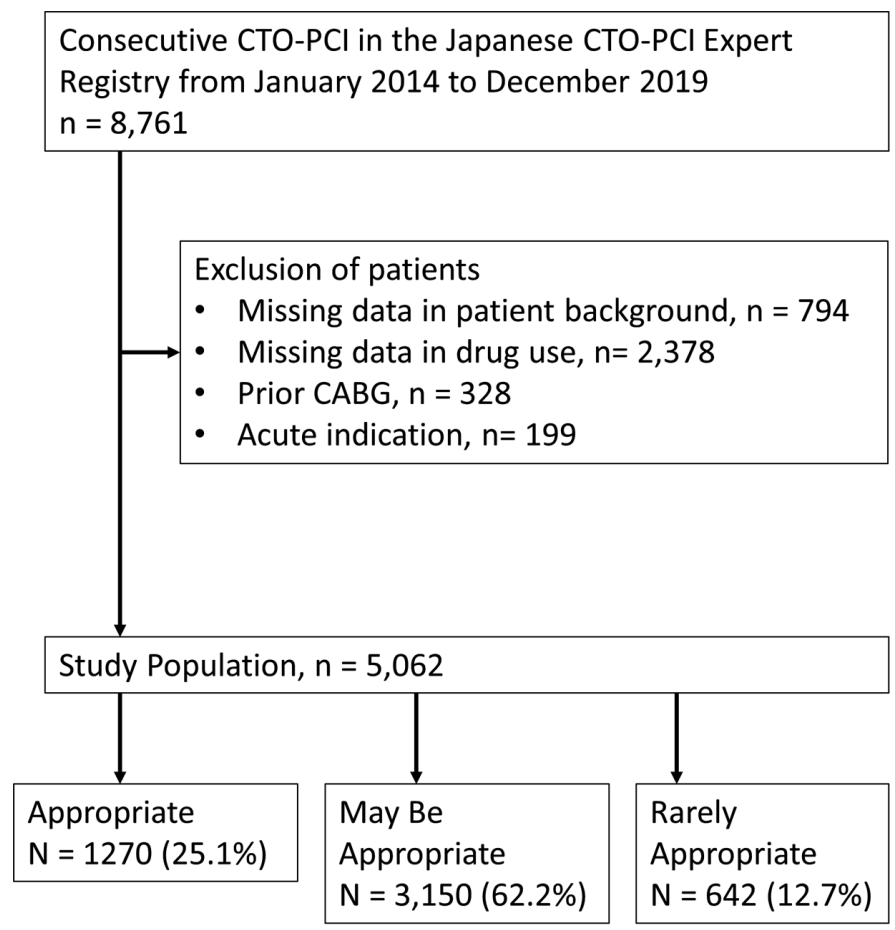

Figure 1. Study flowchart. CTO, chronic total occlusion; $\mathrm{PCl}$, percutaneous coronary intervention; $\mathrm{CABG}$, coronary artery bypass grafting.

Total Occlusion [DECISION-CTO] trial) including 834 patients with a CTO lesion showed that CTO-PCI did not decrease the rate of a composite of death, myocardial infarction, stroke, or any revascularization compared to optimal medical therapy alone, whereas the study was limited by low power for clinical endpoints and high crossover rates between groups. ${ }^{11}$ Therefore, appropriate patient selection for CTO-PCI has become even more important.

However, the appropriateness of CTO-PCI and its impact on prognosis in real-world practice are still unclear. Therefore, we conducted this cross-sectional study to elucidate the appropriateness of CTO-PCI and its prognostic impact on patients selected from a Japanese CTO registry.

\section{Methods}

\section{Data Source}

This study is a sub-study of the Japanese CTO-PCI Expert Registry, which is a prospective, non-randomized registry enrolling consecutive patients undergoing CTO-PCI performed by Japanese CTO operators. This registry's design and enrollment status have been reported previously. ${ }^{\mathbf{4 1 2}}$ The registry data are managed by the secretariat (Clinical Research Center, Kurashiki Central Hospital, Ohara Healthcare Foundation, Okayama, Japan). Diagnostic coronary angiograms, computed tomography images of the coronary arteries before the interventions, and intravascular ultrasound images obtained during the interventions are sent to an independent core laboratory (Cardiovascular Imaging Center, Aichi, Japan) for further analysis; the core laboratory calculated the SYNergy Between PCI with TAXus and Cardiac Surgery (SYNTAX) score. The Japanese Board of CTO Interventional Specialists was established in July 2013 and with certified 46 highly experienced Japanese specialists by the end of 2019 . The requirements for certification were that the specialist performed more than 50 CTO-PCIs per year and that they had performed more than 300 CTO-PCIs in total. The certified specialists are required to enroll all consecutive CTO-PCI cases into the registry. The planned patient enrollment period is from January 2014 to December 2022, and the clinical follow up will continue until December 2027. Written informed consent was obtained from all patients, the sub-study was approved by the ethics committee of Kyoto University (R1701) and followed the principles of the Declaration of Helsinki.

\section{Study Population}

We included consecutive patients who underwent CTOPCI between January 2014 and December 2019. The following patients were excluded: those having a missing variable in the patient background $(n=794,21.5 \%)$ or drug use $(n=2,398,64.8 \%)$, those with a history of prior coronary artery bypass graft $(\mathrm{CABG})$ surgery $(\mathrm{n}=328,8.9 \%)$, and those who underwent PCI for acute indications (acute myocardial infarction and unstable angina including Canadian Cardiovascular Society class 4; $n=199,5.4 \%$ ).

\section{Definitions}

CTO was defined as the presence of Thrombolysis in Myocardial Infarction flow grade 0 within an occluded arterial segment for $\geq 3$ months. We classified "appropriateness" according to the key variables based on 2017 AUC for Coronary Revascularization in Patients with SIHD. 5 One vessel disease was classified according to the following variables: whether proximal left anterior descending (LAD) or proximal left dominant left circumflex (LCX) was involved, non-invasive risk stratification, anginal symptoms, and the number of antianginal medications at discharge. Two vessel 
Table 1. Characteristics of Patients Undergoing CTO-PCls Stratified by Appropriateness

\begin{tabular}{|c|c|c|c|c|c|}
\hline & $\begin{array}{c}\text { Total } \\
(n=5,062)\end{array}$ & $\begin{array}{l}\text { Appropriate } \\
(n=1,270)\end{array}$ & $\begin{array}{c}\text { May be } \\
\text { appropriate } \\
(n=3,150)\end{array}$ & $\begin{array}{c}\text { Rarely } \\
\text { appropriate } \\
(\mathrm{n}=642)\end{array}$ & $P$ value \\
\hline Age, years & $67.3(11.2)$ & $67.3(11.5)$ & $67.3(11.1)$ & $67.6(11.3)$ & 0.81 \\
\hline BMI, kg/m² & $24.8(3.8)$ & $25.0(3.9)$ & $24.8(3.7)$ & $24.8(4.0)$ & 0.12 \\
\hline LVEF, \% & $55.0(13.0)$ & $52.1(13.7)$ & $54.7(12.7)$ & $62.5(9.9)$ & $<0.001$ \\
\hline eGFR, mL/min/1.73 $\mathrm{m}^{2}$ & $64.0(21.5)$ & $62.8(21.7)$ & $64.3(21.3)$ & $65.0(22.1)$ & 0.04 \\
\hline Male sex & $4,308(85.1)$ & $1,067(84.0)$ & $2,699(85.7)$ & $542(84.4)$ & 0.32 \\
\hline Current smoker & $789(17.6)$ & $223(17.6)$ & $564(17.9)$ & $102(15.9)$ & 0.008 \\
\hline Hypertension & $3,865(76.4)$ & $1,053(82.9)$ & $2,347(74.5)$ & $465(72.4)$ & $<0.001$ \\
\hline Dyslipidemia & $4,119(81.4)$ & $1,026(80.8)$ & $2,584(82.0)$ & $509(79.3)$ & 0.22 \\
\hline Diabetes & 2,218 (43.8) & $535(42.1)$ & $1,409(44.7)$ & $274(42.7)$ & 0.24 \\
\hline History of MI & $2,285(45.1)$ & $616(48.5)$ & $1,495(47.4)$ & $174(27.1)$ & $<0.001$ \\
\hline Prior PCl & $3,269(64.6)$ & $829(65.3)$ & $2,047(65.0)$ & 393 (61.2) & 0.16 \\
\hline Peripheral artery disease & $527(10.4)$ & $105(8.3)$ & $320(10.2)$ & $102(15.9)$ & $<0.001$ \\
\hline Aspirin & $5,009(99.0)$ & $1,253(98.7)$ & $3,118(99.0)$ & $638(99.4)$ & 0.34 \\
\hline P2Y12 inhibitors & $4,981(98.4)$ & $1,247(98.2)$ & $3,105(98.6)$ & $629(98.0)$ & 0.43 \\
\hline Anticoagulants & $425(8.4)$ & $139(11.0)$ & $236(7.5)$ & $50(7.8)$ & $<0.001$ \\
\hline ACE inhibitors & 973 (19.2) & $282(22.2)$ & $603(19.1)$ & $88(13.7)$ & 0.007 \\
\hline ARBs & $2,179(43.1)$ & $569(44.8)$ & $1,332(42.3)$ & $278(43.3)$ & 0.31 \\
\hline Statins & $4,188(82.7)$ & $1,050(82.7)$ & $2,618(83.1)$ & $520(81.0)$ & 0.43 \\
\hline$\beta$-blockers & $2,486(49.1)$ & $914(72.0)$ & $1,341(42.6)$ & $231(36.0)$ & $<0.001$ \\
\hline Calcium channel blockers & $1,954(38.6)$ & $706(55.6)$ & $1,015(32.2)$ & $233(36.3)$ & $<0.001$ \\
\hline Nitrates & $1,088(21.5)$ & 477 (37.6) & 499 (15.8) & $112(17.5)$ & $<0.001$ \\
\hline PPIs & $4,153(82.0)$ & $1,033(81.3)$ & $2,602(82.6)$ & $518(80.7)$ & 0.39 \\
\hline \multicolumn{6}{|l|}{ Key variables } \\
\hline Anginal symptoms & & & & & $<0.001$ \\
\hline No symptoms & $2,492(49.2)$ & $260(20.5)$ & $1,748(55.5)$ & $484(75.4)$ & \\
\hline CCS class I & $944(18.7)$ & $382(30.1)$ & $504(16.0)$ & $58(9.0)$ & \\
\hline CCS class II & $1,509(29.8)$ & $579(45.6)$ & $841(26.7)$ & $89(13.9)$ & \\
\hline CCS class III & $117(2.3)$ & $49(3.9)$ & $57(1.8)$ & $11(1.7)$ & \\
\hline No. of antianginal drugs & & & & & $<0.001$ \\
\hline 0 & $1,136(22.4)$ & $33(2.6)$ & $862(27.4)$ & $241(37.5)$ & \\
\hline 1 & $2,523(49.8)$ & $494(38.9)$ & $1,779(56.5)$ & $250(38.9)$ & \\
\hline 2 & $1,204(23.8)$ & $626(49.3)$ & $451(14.3)$ & $127(19.8)$ & \\
\hline 3 & $199(3.9)$ & $117(9.2)$ & $58(1.8)$ & $24(3.7)$ & \\
\hline $\begin{array}{l}\text { High or intermediate risk in } \\
\text { non-invasive tests }\end{array}$ & $3,261(64.4)$ & $991(78.0)$ & $2,203(69.9)$ & $67(10.4)$ & $<0.001$ \\
\hline No. of diseased vessels & & & & & $<0.001$ \\
\hline 1 & $2,230(44.1)$ & $456(35.9)$ & $1,378(43.8)$ & $396(61.7)$ & \\
\hline 2 & $1,646(32.5)$ & $626(49.3)$ & $865(27.5)$ & $155(24.1)$ & \\
\hline 3 & $1,040(20.6)$ & $181(14.3)$ & $859(27.3)$ & $0(0)$ & \\
\hline LMT involvement & $146(2.9)$ & $7(0.55)$ & $48(1.5)$ & $91(14.2)$ & \\
\hline Proximal LAD involvement & $1,675(33.1)$ & $472(37.2)$ & $1,123(35.7)$ & $80(12.5)$ & $<0.001$ \\
\hline
\end{tabular}

Continuous variables (Age, BMI, LVEF, and eGFR) are represented as mean (SD), and categorical variables as $n$ (\%). ACE, angiotensinconverting enzyme; ARB, angiotensin receptor blocker; BMI, body mass index; CCS, Canadian Cardiovascular Society; CTO, chronic total occlusion; eGFR, estimated glomerular filtration rate; LAD, left anterior descending artery; LMT, left main trunk; LVEF, left ventricular ejection fraction; MI, myocardial infarction; $\mathrm{PCl}$, percutaneous coronary intervention; PPI, proton pump inhibitor.

disease was classified according to the following variables: whether proximal LAD was involved, diabetes mellitus, non-invasive risk stratification, anginal symptoms, and the number of antianginal medications at discharge. Three vessel disease was classified according to the following variables: SYNTAX score, diabetes mellitus, non-invasive risk stratification, anginal symptoms, and the number of antianginal medications at discharge. Unprotected left main trunk (ULMT) disease was classified according to the following variables: SYNTAX score, bifurcation involvement, concurrent multivessel disease, anginal symptoms, and the number of antianginal medications at discharge. High or intermediate non-invasive risk stratification was determined if the patient satisfied at least one of the following criteria: a positive stress electrocardiography (ECG) test result, left ventricular ejection fraction (LVEF) $<50 \%$, and non-normal wall motion at the CTO site. The lesion was deemed as intermediate or high-disease complexity if the 


\begin{tabular}{|c|c|c|}
\hline Indication* & Description & \\
\hline 1 & $\begin{array}{l}1 \text {-vessel disease, no proximal LAD disease, low-risk findings on non-invasive testing, and asymptomatic } \\
\text { or symptomatic but not on antianginal therapy }\end{array}$ & $396(61.7)$ \\
\hline 7 & 2-vessel disease, no proximal LAD disease, low-risk findings on non-invasive testing, and asymptomatic & $155(24.1)$ \\
\hline 29 & Left main disease, bifurcation involvement, SYNTAX score $>22$ & $91(14.2)$ \\
\hline
\end{tabular}

These reasons comprised $100 \%$ of all "rarely appropriate" PCls. Data are presented as $\mathrm{n}(\%)$. *Scenario numbers reported by Patel et al. ${ }^{5}$ SYNTAX, SYNergy Between PCI With TAXus and Cardiac Surgery. Other abbreviations as in Table 1.

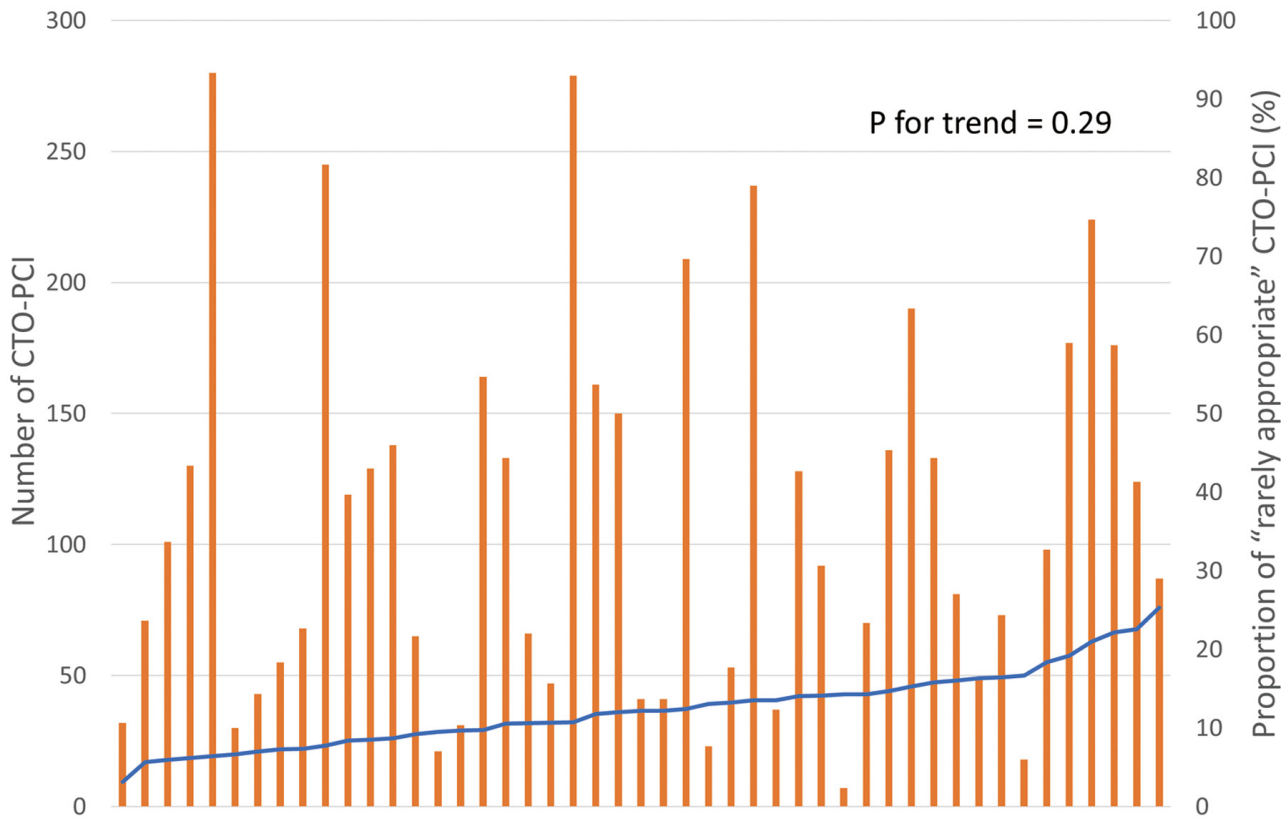

Figure 2. Proportion of "rarely appropriate" CTO-PCI procedures performed by the operators. The vertical bar graph denotes the total number, and the line graph denotes the proportion of "rarely appropriate" CTO-PCI procedures by each operator. CTO-PCI, chronic total occlusion-percutaneous coronary intervention.

patient had 3 vessel or ULMT disease and the SYNTAX score was $>22$. As $4,309(85.1 \%)$ patients did not undergo a stress ECG and no other non-invasive stress test was recorded in the registry, patients who did not undergo a stress ECG and those with undeterminable stress test results were classified as having a negative stress ECG test result when invasive risk stratification was determined.

\section{Statistical Analysis}

The baseline characteristics of patients undergoing CTOPCI were compared according to the appropriateness category. Continuous variables were evaluated using analysis of variance and categorical variables using the chi-squared test. The Cochran-Armitage test was used to evaluate the trend of the proportion of "rarely appropriate" PCIs by each operator or calendar year. Bivariable and multivariable logistic regression analyses were performed to evaluate the association between the appropriateness of CTO-PCI and major adverse cardiovascular and cerebrovascular events (MACCE), and odds ratios (OR) and 95\% confidence intervals (CIs) were presented. In the multivariable model, the variables included "may be appropriate" or "rarely appropriate" PCI vs. "appropriate" PCI, age, LVEF, the estimated glomerular filtration rate (eGFR), male sex, current smoker, history of myocardial infarction (MI), hypertension, dyslipidemia, diabetes mellitus, peripheral artery disease, the Canadian Cardiovascular Society class, and the number of diseased vessels. Variables were selected according to clinical importance, and no statistical variable selection method was applied. Additionally, one of the major updates that was incorporated in the 2017 AUC was the addition of CT and fractional flow reserve-based approach towards PCIs. To elucidate whether coronary computed tomography angiography (CCTA) use before CTO-PCI was associated with the risk of "rarely appropriate" CTO-PCI, we conducted another bivariable and multivariable logistic regression analysis, and OR and 95\% CIs were presented. In this multivariable model, variables included CCTA use, anginal symptom (symptomatic vs. asymptomatic), high or intermediate non-invasive risk, the number of antianginal medications at discharge, and the number of diseased vessels. Covariates were selected according to the previous study that evaluated the association between CCTA use and the appropriateness of PCI. ${ }^{8}$ 
100

80

P for trend $=0.89$

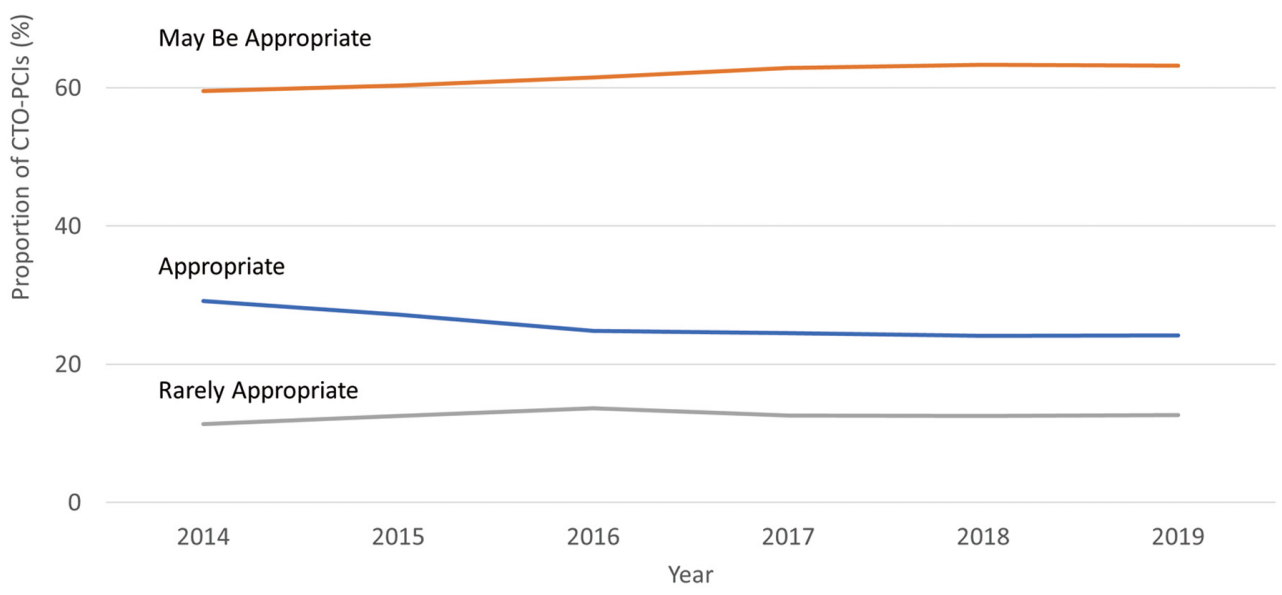

Figure 3. Proportion of "appropriate", "may be appropriate", and "rarely appropriate" CTO-PCI cases from 1 July 2014 to 31 December 2019. CTO-PCI, chronic total occlusion-percutaneous coronary intervention.

\begin{tabular}{|lccccc|}
\hline Table 3. In-Hospital Outcomes & $\begin{array}{c}\text { Total } \\
(\mathbf{n = 5 , 0 6 2 )}\end{array}$ & $\begin{array}{c}\text { Appropriate } \\
(\mathbf{n = 1 , 2 7 0 )}\end{array}$ & $\begin{array}{c}\text { May be } \\
\text { appropriate } \\
(\mathbf{n = 3 , 1 5 0 )}\end{array}$ & $\begin{array}{c}\text { Rarely } \\
\text { appropriate } \\
(\mathbf{n = 6 4 2})\end{array}$ & $\begin{array}{c}\mathbf{P} \text { value } \\
\text { Technical success }\end{array}$ \\
MACCE & $4,725(93.4)$ & $1,188(93.8)$ & $2,935(93.2)$ & $602(93.9)$ & 0.69 \\
Death & $71(1.4)$ & $19(1.5)$ & $46(1.5)$ & $6(0.9)$ & 0.56 \\
Myocardial infarction & $4(0.08)$ & $1(0.08)$ & $3(0.1)$ & $0(0)$ & 0.73 \\
Stent thrombosis & $55(1.1)$ & $15(1.2)$ & $34(1.1)$ & $6(0.9)$ & 0.88 \\
Stroke & $2(0.04)$ & $0(0)$ & $2(0.06)$ & $0(0)$ & 0.54 \\
Cardiac tamponade & $10(0.2)$ & $3(0.24)$ & $7(0.22)$ & $0(0)$ & 0.48 \\
Puncture site complication & $129(2.6)$ & $41(3.2)$ & $75(2.4)$ & $13(2.0)$ & 0.18 \\
Emergency revascularizations & $75(1.5)$ & $19(1.5)$ & $41(1.3)$ & $15(2.3)$ & 0.14 \\
\hline
\end{tabular}

Data are presented as $\mathrm{n}(\%)$. MACCE, major cardiovascular and cerebrovascular events.

Finally, we conducted a sensitivity analysis to account for the missing variables in drug use. In sensitivity analyses, patients with missing variables in drug use were included, and the number ( $\%$ ) of appropriateness was recalculated. In the best-case scenario, missing drug use was classified positive, and in the worst-case scenario, this variable was classified negative. Data were analyzed using SAS 9.4 (Cary, NC, USA). All P values were 2-sided, and significance was defined as $\mathrm{P}<0.05$ for all analyses.

\section{Results}

Of the 8,761 patients who underwent PCI for CTO lesions between January 2014 and December 2019, we excluded 3,699 patients who met the exclusion criteria. In total, we investigated 5,062 patients in this study. Of these, 1,270 $(25.1 \%)$ were rated as "appropriate," 3,150 (62.2\%) as "may be appropriate", and $642(12.7 \%)$ as "rarely appropriate" PCI cases (Figure 1).

Table 1 summarizes the clinical characteristics of the patients undergoing CTO-PCI. The mean age was 67.3 years (standard deviation, 11.2), and 4,308 patients (85.1\%) were men. Compared to the procedures classified as "appropriate" or "may be appropriate", "rarely appropriate" PCI procedures were more likely to be performed in patients with single-vessel coronary artery stenosis or ULMT disease, and cases were less likely to involve proximal LAD artery stenosis (Table 1).

The most frequent clinical indications for "rarely appropriate" PCI procedures are outlined in Table 2 . All "rarely appropriate" CTO-PCI procedures were classified into 3 indications. Among patients who were classified as "rarely appropriate", 396 (61.7\%) patients had 1-vessel disease, no proximal LAD disease, low-risk findings on non-invasive 


\begin{tabular}{|c|c|c|c|c|}
\hline & \multicolumn{2}{|c|}{ Bivariable model } & \multicolumn{2}{|c|}{ Multivariable model } \\
\hline & OR $(95 \% \mathrm{Cl})$ & $P$ value & OR (95\% Cl) & $P$ value \\
\hline Appropriate & Reference & & Reference & \\
\hline May be appropriate & $0.98(0.57-1.67)$ & 0.93 & $0.97(0.53-1.76)$ & 0.92 \\
\hline Rarely appropriate & $0.62(0.25-1.56)$ & 0.31 & $0.65(0.22-1.90)$ & 0.43 \\
\hline Age & $1.02(1.00-1.04)$ & 0.11 & $1.02(0.99-1.04)$ & 0.23 \\
\hline LVEF & $0.99(0.97-1.01)$ & 0.27 & $1.00(0.98-1.02)$ & 0.67 \\
\hline eGFR & $1.00(0.99-1.01)$ & 0.51 & $1.00(0.99-1.01)$ & 0.74 \\
\hline Male sex & $0.65(0.37-1.15)$ & 0.14 & $0.69(0.38-1.26)$ & 0.22 \\
\hline Current smoker & $0.86(0.45-1.64)$ & 0.64 & $0.95(0.49-1.85)$ & 0.87 \\
\hline History of MI & $1.49(0.93-2.39)$ & 0.10 & $1.48(0.89-2.49)$ & 0.13 \\
\hline Hypertension & $1.70(0.89-3.24)$ & 0.11 & $1.63(0.85-3.15)$ & 0.14 \\
\hline Dyslipidemia & $1.25(0.66-2.39)$ & 0.50 & $1.24(0.64-2.39)$ & 0.52 \\
\hline Diabetes & $1.40(0.88-2.24)$ & 0.16 & $1.34(0.83-2.15)$ & 0.23 \\
\hline Peripheral artery disease & $1.42(0.72-2.79)$ & 0.31 & $1.36(0.68-2.72)$ & 0.38 \\
\hline \multicolumn{5}{|l|}{ CCS class } \\
\hline No angina & Reference & & Reference & \\
\hline 1 & $0.80(0.41-1.59)$ & 0.28 & $0.79(0.39-1.62)$ & 0.28 \\
\hline 2 & $0.96(0.56-1.66)$ & 0.61 & $0.98(0.55-1.79)$ & 0.74 \\
\hline 3 & $1.80(0.55-5.92)$ & 0.26 & $1.68(0.55-1.79)$ & 0.32 \\
\hline \multicolumn{5}{|c|}{ Number of diseased vessels } \\
\hline 1 & Reference & & Reference & \\
\hline 2 & $1.16(0.66-2.02)$ & 0.54 & $1.11(0.63-1.97)$ & 0.53 \\
\hline 3 & $1.44(0.78-2.62)$ & 0.67 & $1.28(0.69-2.37)$ & 0.97 \\
\hline LMT & $1.71(0.51-5.71)$ & 0.54 & $1.95(0.54-7.03)$ & 0.49 \\
\hline
\end{tabular}

OR, odds ratio; $\mathrm{Cl}$, confidence interval. Other abbreviations as in Table 1.

testing, and were asymptomatic or symptomatic but not on antianginal therapy, whereas $155(24.1 \%)$ patients had 2-vessel disease, no proximal LAD disease, low-risk findings on non-invasive testing, and were asymptomatic (Table 2). Among 91 patients with ULMT disease, bifurcation involvement, and a SYNTAX score > 22, $29(29.6 \%)$ patients had a SYNTAX score between 23 and 32, and 64 $(65.3 \%)$ patients had a SYNTAX score $\geq 33$.

The proportion of "rarely appropriate" procedures differed substantially from operator to operator, varying from $3.1 \%$ to $25.3 \%$; a non-significant trend was observed between appropriateness and the total number of PCI procedures ( $P$ for trend $=0.29$ ) (Figure 2$)$. Additionally, the trend of appropriateness did not change during the study period $(\mathrm{P}$ for trend=0.89; Figure 3 ).

The in-hospital outcomes are listed in Table 3. Technical success was achieved in 4,725 (93.4\%) of cases, and MACCEs were observed in $71(1.4 \%)$ patients. No significant difference was observed among the in-hospital outcomes according to appropriateness (Table 3). The results of bivariate and multivariable logistic regression for MACCE are listed in Table 4. Multivariable logistic regression analysis showed that neither the "may be appropriate" (OR, 0.97; 95\% CI, 0.53-1.76; $\mathrm{P}=0.92$ ) nor the "rarely appropriate" (OR, 0.65; 95\% CI, 0.22-1.90; P=0.43) CTO-PCI cases were associated with MACCE (Table 4).

In this study, 2,990 (59.1\%) patients underwent CCTA before CTO-PCI, and the proportion did not change during the study period ( $\mathrm{P}$ for trend=0.76). In the logistic regression analysis, CCTA use was associated with a higher risk of "rarely appropriate" CTO-PCI in the bivariate analysis (OR, 1.22; 95\% CI, 1.03-1.45; $\mathrm{P}=0.02)$; however, the asso- ciation was not statistically significant in the multivariable analysis (OR, 1.05; 95\% CI, 0.8-1.38; $\mathrm{P}=0.70)$.

The sensitivity analyses included 7,143 patients. In the best-case scenario, 2,175 (30.5\%) were rated as "appropriate", 4,125 (57.8\%) as "may be appropriate", and 843 $(11.8 \%)$ as "rarely appropriate" PCI cases. In the worstcase scenario, 1,429 (20.0\%) were rated as "appropriate", $4,744(66.4 \%)$ as "may be appropriate", and $970(13.6 \%)$ as "rarely appropriate" PCI cases.

\section{Discussion}

In this large registry of patients undergoing CTO-PCI performed by Japanese expert operators, $20 \%$ of cases were rated as "appropriate", $66 \%$ as "may be appropriate", and $15 \%$ as "rarely appropriate". The proportion of "appropriate" cases was lower, but the proportion of "rarely appropriate" PCI cases was comparable to that observed in previous reports. ${ }^{\mathbf{6 , 9}}$ In addition, the appropriateness of CTO-PCI was not associated with in-hospital outcomes, including MACCE.

To our knowledge, this is the largest study investigating the appropriateness of CTO-PCI, although Inohara et $\mathrm{al}^{\mathbf{1 3}}$ analyzed 500 CTO-PCI procedures to compare the appropriateness of CTO vs. non-CTO-PCI in Japanese realworld practice. The present study included a larger number of patients than previous studies, and the results should therefore have greater generalizability.

The indications for CTO-PCI should be considered as well or more stringently than those for non-CTO-PCI because CTO-PCI is highly complex, has a lower technical success rate, has a higher complication rate, and necessi- 
tates greater use of contrast media and increased radiation exposure than non-CTO-PCI. ${ }^{4}$ In this study, patients with the following conditions were classified as "rarely appropriate": those with 1 or 2-vessel disease without proximal LAD involvement and receiving the maximum number of $(\geq 2)$ antianginal medications and those with ULMT lesions involving the bifurcation and a SYNTAX score $>22$.

In the present study, $\geq 80 \%$ of the "rarely appropriate" PCIs were conducted for 1- or 2-vessel disease, without proximal LAD disease, with minimal symptoms, suboptimal antianginal therapy, and low-risk findings on noninvasive testing. The results of cases involving 1- or 2-vessel disease were similar to those of previous studies. In past studies involving not only CTO-PCI but PCI in general, $71-91 \%$ of inappropriate PCIs were conducted for $1-$ or 2-vessel disease. ${ }^{6,79}$ Similarly, the rate of "rarely appropriate" PCIs was substantially different among operators, as past studies showed that the range of inappropriate PCI was $0-1 \%$ to $35-55 \%$ among institutions.

Conversely, our study included a large number of patients with ULMT disease who were rated as "rarely appropriate", which was different from the findings of earlier studies. The high rate of "rarely appropriate" ratings in patients with ULMT disease implies the difference of guideline and clinical practice between countries. National Cardiovascular Data Registry CathPCI Registry in the US reported that PCI was performed in only $4.3 \%$ of ULMT disease cases between 2004 and 2008.14 Conversely, the CREDO-Kyoto $\mathrm{PCI} / \mathrm{CABG}$ registry in Japan also reported that $36.3 \%$ of revascularizations for ULMT disease was by PCI between 2005 and 2007.15 The discrepancy showed that PCI for ULMT disease is more common in Japan than in the US. In the present study, 29/98 (29.6\%) patients with ULMT disease with bifurcation and a SYNTAX score of 23-32 were classified to "rarely appropriate". However, the European Society of Cardiology/European Association for Cardio-Thoracic Surgery guideline advocates PCI for ULMT disease with a SYNTAX score 23-32 in class IIa, although AUC 2017 rated these PCIs as "rarely appropriate".16

A previous study from the United States showed that the rate of "rarely appropriate" PCI procedures decreased from 2009 to 2014 , but such a trend was not observed in the present study. ${ }^{10}$ In the United States, feedback to the operators and financial incentives reduced the number of "rarely appropriate" PCI cases, but there is no similar feedback or incentive system in place in Japan. ${ }^{7}$ Therefore, a feedback or financial incentive model would also be useful in Japan to decrease the rate of "rarely appropriate" PCI cases.

Our results are consistent with those of previous studies that showed that the appropriateness of PCI was not associated with the prognosis of patients. ${ }^{17,18}$ Similarly, results from the present study are consistent with 2 recent RCTs. As previously described, the DECISION-CTO trial, which randomized 834 patients from Asian countries in a $1: 1$ ratio, showed that CTO-PCI did not decrease the rate of a composite of death, MI, stroke, or any revascularization compared to optimal medical therapy alone. In contrast, the EUROCTO trial (A Randomized Multicenter Trial to Evaluate the Utilization of Revascularization or Optimal Medical Therapy for the Treatment of Chronic Total Coronary Occlusions), which randomized 396 patients from European countries in a 2:1 ratio, showed that CTO-PCI improved the angina frequency and quality of life subscales in the Seattle angina questionnaire. ${ }^{11,19}$ The discrepancy between these 2 RCTs implies that CTO-PCI alleviates anginal symptoms, but does not decrease hard endpoints such as death or MI, as well as non-CTO-PCIs. ${ }^{20-23}$ However, we could not assess the change of anginal symptoms because anginal symptoms after CTO-PCI were not recorded in our registry. Thus, it is necessary to evaluate not only the hard outcomes but also patient-reported quality of life in future studies.

In the present study, the proportion of CCTA use before CTO-PCI was approximately $60 \%$ and was not associated with a higher risk of "rarely appropriate" CTO-PCI in the multivariable logistic regression analysis. In contrast, Inohara et $\mathrm{al}^{\mathbf{8}}$ reported that the CCTA use was associated with fewer stress myocardial perfusion imagings and more "rarely appropriate" non-acute PCI. Similarly, a systematic review of RCTs showed that CCTA increased invasive coronary angiography and revascularization compared to stress testing. ${ }^{24}$ Some patients experienced revascularization after CCTA may have less anginal symptoms and ischemic burden than those after stress testing. Therefore, CCTA use may be associated with "rarely appropriate" PCI in general. However, CCTA is useful for CTO-PCI not only for the diagnosis of the presence or absence of occlusions, but also because it can predict procedural success, assess characteristics of occluded lesions, and real-time fusion imaging. ${ }^{25}$

\section{Study Limitations}

Our study has some limitations. First, we excluded many patients due to missing baseline variables, including patient demographics and medications at discharge. This may induce selection bias if the missing data were not missing completely at random. In addition, because 4,309 (85.1\%) patients did not undergo a stress ECG, we classified these patients as those whose stress test results were negative, although past studies deemed these patients unmappable and were excluded. We might have introduced misclassification bias if a patient was misclassified as "rarely appropriate" or "may be appropriate" even though they had moderate or severe myocardial ischemia. Our registry included only stress ECG results among non-invasive stress tests; stress echocardiography, single photon emission computed tomography, and stress magnetic resonance imaging were not included. Therefore, non-invasive risk stratification was performed according to the stress ECG results, LVEF, and wall motion. If additional non-invasive stress tests had been performed, the appropriateness ratings may have been different. In addition, findings of stress ECGs were recorded simply as positive/negative and detailed information was not available, therefore misclassification was possible. To mitigate the effect of missing information regarding drug use, we conducted sensitivity analyses. In the sensitivity analyses, more patients were classified to "appropriate" in the best-case scenario and "rarely appropriate" in the worst-case scenario than in the primary analysis. "Rarely appropriate" ratings in the best- and worst-case scenarios were very similar to the primary analysis, but a $10 \%$ difference was observed in the "appropriate" rating between the best- and worst-case scenarios. Furthermore, we used the information about antianginal medication at discharge instead of those before CTO-PCI, because the registry did not include the information about drug usage before CTO-PCI. Then, it is possible that some patients might have stopped antianginal medications at discharge. It is necessary to record detailed information pertaining to non-invasive stress testing and antianginal 
medications to minimize the bias due to missing variables and misclassification in future studies. Second, our results may not be generalizable to general clinical practice, including low-volume operators, because our database only included stringently selected operators. Third, our results could not be directly compared with those of previous studies using the 2009 or 2012 AUC because we used the 2017 AUC in the present study. Hannan et $\mathbf{a l}^{\mathbf{2 6}}$ showed that the number of cases rated as "rarely appropriate/inappropriate" were lower in the 2017 AUC than in the 2012 AUC. If the patients in our study were rated according to the 2012 AUC, it is possible that the number of patients rated as "rarely appropriate/inappropriate" would have increased. Finally, the fractional flow reserve value was not recorded in our database; however, this lack of information may not have resulted in bias because all the culprit lesions in this registry were CTO lesions.

\section{Conclusions}

In this study, approximately $13 \%$ of CTO-PCI were rated as "rarely appropriate"; however, the findings in this study should be interpreted with caution because for a large number of patients, there was no information on noninvasive stress testing. It is essential to take greater efforts to assemble more detailed data and decrease the number of "rarely appropriate" cases in daily clinical practice.

\section{Acknowledgments}

The authors are very grateful to the late Dr. Kazuaki Mitsudo for his great efforts with regard to this registry and would like to dedicate this paper to him. The authors would like to thank Drs. Yasumi Igarashi, Tsutomu Fujita, Yoshihito Yamamoto, Kaname Takizawa, Tohru Takahashi, Jiro Ando, Yuichi Noguchi, Yuji Oikawa, Akitsugu Oida, Shinya Okazaki, Yuji Hamazaki, Makoto Muto, Makoto Sekiguchi, Toshiya Muramatsu, Masahisa Yamane, Kazuhiro Ashida, Yasushi Asakura, Yoshiki Uehara, Masakazu Nagaoka, Yuichi Kobori, Kenichiro Shimoji, Yoshiaki Ito, Hisayuki Okada, Osamu Katoh, Etsuo Tsuchikane, Yoshihisa Kinoshita, Kenya Nasu, Fumitaka Hosaka, Kinzo Ueda, Satoru Otsuji, Atsunori Okamura, Takafumi Tsuji, Yoshihiro Takeda, Satoru Sumitsuji, Wataru Nagamatsu, Ryohei Yoshikawa, Kenji Kawajiri, Shigeru Nakamura, Masaki Tanabe, Tomohiro Kawasaki, Koichi Kishi, Yoshisato Shibata, and Takeshi Serikawa for enrolling their patients in the present study.

\section{Sources of Funding}

This research was supported by JSPS KAKENHI (Grant Number JP19K10509).

\section{Disclosures}

K.K. reports personal fees from LEBER. Inc, JMDC, grants from Eisai, Kyowa Hakko Kirin, Shin Nippon Biomedical Laboratories, Sumitomo Dainippon Pharma Co., Ltd., Pfizer Inc, Stella Pharma Corporation, Suntory Beverage \& Food Ltd., Mitsubishi Co., and grants from and stock holding in Real World Data, Co., Ltd. Other authors report no conflicts of interest.

\section{IRB Information}

The study was approved by the ethics committee of Kyoto University (R1701)

\section{References}

1. Jeroudi OM, Alomar ME, Michael TT, Sabbagh AE, Patel VG, Mogabgab O, et al. Prevalence and management of coronary chronic total occlusions in a tertiary veterans affairs hospital. Catheter Cardiovasc Interv 2014; 84: 637-643.

2. Fefer P, Knudtson ML, Cheema AN, Galbraith PD, Osherov $\mathrm{AB}$, Yalonetsky $\mathrm{S}$, et al. Current perspectives on coronary chronic total occlusions. J Am Coll Cardiol 2012; 59: 991 -997.
3. Christofferson RD, Lehmann KG, Martin GV, Every N, Caldwell JH, Kapadia SR. Effect of chronic total coronary occlusion on treatment strategy. Am J Cardiol 2005; 95: 1088-1091.

4. Suzuki Y, Tsuchikane E, Katoh O, Muramatsu T, Muto M, Kishi K, et al. Outcomes of percutaneous coronary interventions for chronic total occlusion performed by highly experienced Japanese specialists: The first report from the Japanese CTO-PCI Expert Registry. JACC Cardiovasc Interv 2017; 10: 2144-2154.

5. Patel MR, Calhoon JH, Dehmer GJ, Grantham JA, Maddox TM, Maron DJ, et al. ACC/AATS/AHA/ASE/ASNC/SCAI/ SCCT/STS 2017 Appropriate use criteria for coronary revascularization in patients with stable ischemic heart disease: A report of the American College of Cardiology Appropriate Use Criteria Task Force, American Association for Thoracic Surgery, American Heart Association, American Society of Echocardiography, American Society of Nuclear Cardiology, Society for Cardiovascular Angiography and Interventions, Society of Cardiovascular Computed Tomography, and Society of Thoracic Surgeons. J Am Coll Cardiol 2017; 69: 2212-2241.

6. Bradley SM, Maynard C, Bryson CL. Appropriateness of percutaneous coronary interventions in Washington State. Circ Cardiovasc Qual Outcomes 2012; 5: 445-453.

7. Hannan EL, Cozzens K, Samadashvili Z, Walford G, Jacobs AK, Holmes DR, et al. Appropriateness of coronary revascularization for patients without acute coronary syndromes. $J \mathrm{Am}$ Coll Cardiol 2012; 59: 1870-1876.

8. Inohara T, Kohsaka S, Miyata H, Ueda I, Ishikawa S, Ohki T, et al. Appropriateness ratings of percutaneous coronary intervention in Japan and its association with the trend of noninvasive testing. JACC Cardiovasc Interv 2014; 7: 1000-1009.

9. Chan PS, Patel MR, Klein LW, Krone RJ, Dehmer GJ, Kennedy $\mathrm{K}$, et al. Appropriateness of percutaneous coronary intervention. JAMA 2011; 306: 53-61.

10. Desai NR, Bradley SM, Parzynski CS, Nallamothu BK, Chan PS, Spertus JA, et al. Appropriate use criteria for coronary revascularization and trends in utilization, patient selection, and appropriateness of percutaneous coronary intervention. JAMA 2015; 314: 2045-2053.

11. Lee SW, Lee PH, Ahn JM, Park DW, Yun SC, Han S, et al. Randomized trial evaluating percutaneous coronary intervention for the treatment of chronic total occlusion. Circulation 2019; 139: $1674-1683$.

12. Tanaka H, Tsuchikane E, Muramatsu T, Kishi K, Muto M, Oikawa $\mathrm{Y}$, et al. A novel algorithm for treating chronic total coronary artery occlusion. J Am Coll Cardiol 2019; 74: 2392-2404.

13. Inohara T, Kohsaka S, Miyata H, Ueda I, Ishikawa S, Ohki T, et al. Real-world use and appropriateness of coronary interventions for chronic total occlusion (from a Japanese multicenter registry). Am J Cardiol 2015; 116: 858-864.

14. Brennan JM, Dai D, Patel MR, Rao SV, Armstrong EJ, Messenger JC, et al. Characteristics and long-term outcomes of percutaneous revascularization of unprotected left main coronary artery stenosis in the United States: A report from the national cardiovascular data registry, 2004 to 2008. J Am Coll Cardiol 2012; 59: $648-654$.

15. Shiomi H, Morimoto T, Furukawa Y, Nakagawa Y, Sakata R, Okabayashi $\mathrm{H}$, et al. Comparison of percutaneous coronary intervention with coronary artery bypass grafting in unprotected left main coronary artery disease: 5 -year outcome from CREDOKyoto PCI/CABG registry cohort-2. Circ J 2015; 79: 1282-1289.

16. Neumann FJ, Sousa-Uva M, Ahlsson A, Alfonso F, Banning AP, Benedetto U, et al. 2018 ESC/EACTS Guidelines on myocardial revascularization. Eur Heart J 2018; 40: 87-165.

17. Barbash IM, Dvir D, Torguson R, Xue Z, Satler LF, Pichard $\mathrm{AD}$, et al. Prognostic implications of percutaneous coronary interventions performed according to the appropriate use criteria for coronary revascularization. Cardiovasc Revasc Med 2013; 14: $316-320$.

18. Seixas AC, Sousa A, de Ribamar Costa J, Costa Moreira A, Costa $\mathrm{R}$, Damiani L. Impact of PCI appropriateness in the long-term outcomes of consecutive patients treated with second-generation drug-eluting stents. J Invasive Cardiol 2017; 29: 290-296.

19. Werner GS, Martin-Yuste V, Hildick-Smith D, Boudou N, Sianos $\mathrm{G}$, Gelev V, et al. A randomized multicentre trial to compare revascularization with optimal medical therapy for the treatment of chronic total coronary occlusions. Eur Heart J 2018; 39: 2484-2493.

20. Boden WE, O'Rourke RA, Teo KK, Hartigan PM, Maron DJ, Kostuk WJ, et al. Optimal medical therapy with or without PCI for stable coronary disease. $N$ Engl J Med 2007; 356: 1503-1516. 
21. Maron DJ, Hochman JS, Reynolds HR, Bangalore S, O'Brien SM, Boden WE, et al. Initial invasive or conservative strategy for stable coronary disease. $N$ Engl J Med 2020; 382: 13951407.

22. Weintraub WS, Spertus JA, Kolm P, Maron DJ, Zhang Z, Jurkovitz C, et al. Effect of PCI on quality of life in patients with stable coronary disease. $N$ Engl J Med 2008; 359: 677-687.

23. Spertus JA, Jones PG, Maron DJ, O'Brien SM, Reynolds HR, Rosenberg Y, et al. Health-status outcomes with invasive or conservative care in coronary disease. $N$ Engl J Med 2020; 382: $1408-1419$.

24. Foy AJ, Dhruva SS, Peterson B, Mandrola JM, Morgan DJ,
Redberg RF. Coronary computed tomography angiography vs functional stress testing for patients with suspected coronary artery disease: A systematic review and meta-analysis. JAMA Intern Med 2017; 177: 1623-1631.

25. Velagapudi P, Abbott JD, Mamas M, Blankstein R, Chatzizisis YS, Brilakis ES, et al. Role of coronary computed tomography angiography in percutaneous coronary intervention of chronic total occlusions. Curr Cardiovasc Imaging Rep 2020; 13: 1-10.

26. Hannan EL, Samadashvili Z, Cozzens K, Berger PB, Chikwe J, Jacobs AK, et al. 2017 versus 2012 appropriate use criteria for percutaneous coronary interventions: Impact on appropriateness ratings. JACC Cardiovasc Interv 2018; 11: 473-478. 